\title{
Chinese herbal medicines with beneficial effects
}

\author{
De-an Guo*, Wenlong Wei, Changliang Yao, Jianqing Zhang, Qirui Bi
}

\begin{abstract}
Among the vast resources of traditional Chinese medicine (TCM) herbal species, only a handful of Chinese herbs are growing in frigid regions or extreme environment but they have a unique property. The most recognizable TCM herb falling in this category is Panax ginseng, which is widely considered the representative tonic herb with oceans of beneficial effects on human health. In this article, we will introduce several typical Chinese herbal medicines with beneficial effects aiming to arouse broader attention from the scientific community to expand the exploration and exploitation on this for their potential applications to meet the increasingly demanding medical needs.
\end{abstract}

\section{Keywords}

traditional Chinese medicine; beneficial effects; panax ginseng; rhodiola rosea; schisandra chinensis

Received 5 March 2021 accepted 15 April 2021
Shanghai Research Centre for Modernization of Traditional Chinese Medicine, National Engineering Laboratory for TCM Standardization Technology, Shanghai Institute of Materia Medica, Chinese Academy of Sciences, 501 Haike Road, Shanghai 201203, China

*Corresponding author Prof. De-an Guo, E-mail: daguo@simm.ac.cn

\section{Introduction}

Traditional Chinese medicine (TCM) has been practiced in China for thousands of years for the treatment of arrays of diseases and plays a pivotal role in maintaining Chinese people's health and well-being, having nurtured and fueled the Chinese civilization and prosperity for the past several millennial historic course. TCM has deeply rooted in the Chinese culture and daily life and slowly immersed into diverse aspects of human life in the big health era. Especially, TCM has aroused greater awareness of international community and witness its gradual global exposure and recognition since the breakthrough of the covid-19 pandemic. To date, there have been over 12807 species were surveyed according to the statistics of 4th national TCM resource census, of which 11146 are herbal drugs, 1581 animal-derived species and 80 minerals. Among them, there are a handful of well-known TCMs, the majority of which are growing in frigid zone (Northeast China) or high-altitude regions (Qinghai-Tibet Plateau). The most of particular TCMs include Panax ginseng, Rhodiola rosea, Schisandra chinensis, Aralia elata, Acanthopanax senticosus, Oplopanax elatus, and Rhaponticum carthamoides, which have been widely used for treating various diseases and strengthening human immunity. This review article will focus on these herbal medicines for their beneficial activity.

Since the introduction of the unique property of TCMs, scientists have identified a few herbal drugs that possess beneficial effects. For instance, Ginseng as a tonic herb can enhance the resistance to cold and stress and normalization of homeostasis in humans. Research focusing on searching herbs with beneficial activity and additional medical benefits. It was found that the herbs are unique from other TCM products in which they possess the ability to restore the equilibrium, modulate nervous and immune system, and maintain the optimal homeostasis. Published studies have documented that particular herbs, as represented by Panax ginseng, can elicit diverse pharmacological effects, such as physical performance enhancing, vitality promoting, anti-stress, anti-aging, immunomodulatory, neuromodulatory, vasomodulatory, antihypertensive, antihypotensive, cardioprotective effects, etc. A recently published article[2] indicated that the special TCMs could be useful in preventing and treating viral infections like COVID-19 at all stages of progression of inflammation, as well as in accelerating the recovery of the organism by improving innate and adaptive immunity, anti-inflammatory activity, detoxification and repair of oxidative stress-induced damage in compromised cells, direct antiviral effects via inhibiting viral docking or replication, and the quality of life during convalescence. These data strongly suggest that the TCM from special growth environment could be used for combating COVID-19.

In an attempt to identify biomarkers for characterizing the beneficial effects of plant extracts, Asea et al.[3] demonstrated that a fixed combination of substances derived from Rhodiola rosea, Schisandra chinensis and several other herbs stimulated the expression and release of neuropeptide Y (NPY) and molecular chaperone Hsp72 from isolated human neuroglia cells. They 
further uncovered that ADAPT-232 induced release of heat shock protein Hsp70 was mediated by NPY, suggesting the existence of the NPY-mediated signaling pathway for Hsp72 release into the blood circulation system. They concluded based on their findings that NPY and Hsp70 could be used as molecular biomarkers and objective criteria for the identification and characterization of particular herbs. Here we are giving brief descriptions on the representative Chinese herbal drugs known to have beneficial effects to uncover for their additional health benefits.

\section{Panax ginseng}

Panax ginseng (known as Asian ginseng) has been widely used as an herbal remedy for various disorders and general wellbeing with a long history ever since it was recorded in Shen Nong's Herbal Classic and regarded as one of the most precious medicinal herbs owing to its remarkable tonic effects. In 1969, Brekhman and Dardymov provided the experimental evidence for the nonspecific and tonic effects of $P$. ginseng, they described that the non-toxic plants or other substances could help increase body's resistance to stress[1]. Since then, numerous studies have been published on the effects of Panax ginseng against inflammation, allergy, hypertensive, diabetes, tumor, obesity, and memory impairment[4-6]. Ginsenosides have been proved to be the major bioactive components of ginseng[7], including two major groups based on their differential chemical structures: protopanaxadiol (Rb1, Rb2, Rc, Rd, etc.) and protopanaxatriol (Rg1, Re, Rf, etc.). Ginseng displayed obvious anti-fatigue and anti-stress efficiency. It was reported that ginseng prolonged swimming time, improved exercise capability, and promoted recovery from fatigue in a forced swimming experiment[8]. Ginseng was also found to inhibit $\mathrm{Ca}^{2+}$ channels in sensory neurons related to the pain transmission. It possesses antioxidation effect through scavenging hydroxyl radical and eliminating damaged DNA[6]. Moreover, ginseng can ameliorate the symptoms of Alzheimer's disease and improve cognitive functions. Many of the therapeutic effects of ginseng is related to its anti-inflammatory property. It was demonstrated that ginseng extract markedly inhibited the expression of iNOS, MMP-9 and proinflammatory cytokines in LPS-stimulated microglial cells, and in mice subjected to systematic administration of LPS[9]. The anti-inflammatory effects of ginseng extracts have been verified with purified ginsenosides, such as Rb1, Rb2, Rd, Re, Rg1, Rg3, $\mathrm{Rg} 5$, Rh1 and Rh2[10]. In addition, ginseng is also widely used in cancer treatment, and multiple studies have associated the consumption of ginseng with cancer prevention and treatment, as well as improved quality-of-life during cancer therapy. Ginsenoside Rg3 isolated from red ginseng showed a remarkable anti-tumor effect and was developed into Shenyi capsule. Furthermore, ginseng can regulate and help maintain normal blood pressure with both antihypertensive and antihypotensive properties. On one hand, the antihypertensive effects of ginseng can be ascribed to its ability to promote vascular endothelial cell-derived NO secretion via the conversion of L-arginine to L-citrulline by eNOS. On the other hand, its antihypotensive effects may be realized via activating vasoconstrictors, i.e. endothelin-1 and angiotensinII. The diverse activities of ginseng may well be related to its beneficial effects, which deserves thorough investigations.

\section{Rhodiola rosea}

Rhodiola rosea (also named "roseroot" or "golden root"), primarily growing in the sandy soil in high-altitude regions of Asia, Europe and Northern Hemisphere, such as Altai Mountains, Iceland, British Isles, has been widely used[11]. In addition to R. rosea, there are over 95 species in the genus Rhodiola and 20 of them have been reported to produce the medicinal effects[12]. Of the Rhodiola species, R. rosea has been extensively explored on its beneficial effects. R. rosea improves the physiological functionalities of the central nervous system, cardiovascular system, endocrine system, and reproductive system[13]. Of note, $R$. rosea relieves fatigue and weakness subsequent to intense physical and psychological stress, enhances learning and memory function, cognition, endurance performance, athletic ability, as well as mood disorders and mental fatigue[11,13]. Also eminent is that $\mathrm{R}$. rosea can produce immune-enhancing, anti-oxidant, and anti-inflammatory effects and suppress the proliferation of cancer cells[14]. At least 140 compounds have been identified in $\mathrm{R}$. rosea extract, and they may have medicinal properties, of which salidroside, p-tyrosol, rosavin, pyridate, rhodiosin, and rhodionin are the most unique active components in the Rhodiola plants[13,15]. R. rosea has been recorded in the pharmacopoeias or herbal drug lists of several countries, and its extracts have been widely used as immune enhancers and for other therapeutic aspects throughout the United States, Europe and Asia, in the forms of Rhodiola capsule, Rhodiola tables and Rhodiola oral liquid[16-17].

\section{Schisandra chinensis}

Schisandra chinensis, named "Wuweizi" in Chinese, belongs to the family of Magnoliaceae and is mainly distributed in wet and humus-rich soil in northeastern China, Korea, Japan, and in the eastern part of Russia[18]. S. chinensis is mainly used in the former Soviet Union with beneficial effects on respiratory failure, cardio-vascular disease, body fatigue and weakness, excessive sweating, and insomnia. In addition, S. chinensis is also reckoned as a tonic agent with hunger reducing, fatigue relieving, aging delaying, vitality enhancing, and mental health improving activities[2, 18-19]. Of particular interesting is that $\mathrm{S}$. chinensis possesses anti-diabetic, anti-obesity, and anti-aging actions as indicated by its actions against skin photoaging, osteoarthritis, 
sarcopenia, senescence, and mitochondrial dysfunction, as well as the improvement of physical endurance and cognitive/behavioral function[20]. Additionally, it can also possess anti-stress, antiinflammatory, antioxidative, detoxification, immunostimulant, cardiovascular and skin-protective properties[18, 21]. These activities are mainly conferred by dibenzocyclooctadiene lignans, which are often referred to as "Schisandra lignans" owing to their unique complex structures. Among these lignans, schisandrin, schisandrin A, schisanhenol, deoxyschisandrin, and gomisin A are the dominant ones[20].

\section{Acanthopanax senticosus}

Acanthopanax senticosus is a medicinal plant of Araliaceae family, which possesses beneficial effects on immune, hormone secretion, central nervous and cardiovascular systems to enhance body's defense system and reduce the damage caused by stress[22-23]. The roots and stems of $A$. senticosus have long been used as functional food and herbal medicine for nourishing Qi to invigorate spleen, tonify kidney and keep tranquilization[24]. Chemical investigation revealed that flavonoids, terpenes, phenylpropanoids and polysaccharides are the major constituents of $A$. senticosus[22]. Pharmacological studies proved that $A$. senticosus could improve learning ability and central nervous system function in irradiated mice through preventing nerve cell swelling, protein loss, and necrotic tissue liquefaction in irradiated mouse brain[25]. The saponins isolated from the herb exert favorable effects on cardiovascular and cerebrovascular systems, decrease the size of myocardial infarction, ameliorate ventricular remodeling, attenuate arrhythmia, and ameliorate ischemicreperfusion injury[26-31]. A. senticosus polysaccharide improves lymphocyte proliferation with increased proportion of $\mathrm{CD}^{4+}$ and $\mathrm{CD}^{8+} \mathrm{T}$ lymphocytes and the levels of interferon gamma and IL-2 in cyclophosphamide-treated chickens compared with non-treated control littermates[32].

\section{Aarlia elata var. mandshurica}

Aarlia elata var. mandshurica (AEM) is another medicinal plant from the Araliaceae family, which has been widely used in China, Korea, and Japan. The AEM saponins and polysaccharides are the main bioactive components, and they possess antitumor and cardioprotective effects[33]. The total saponins significantly increase the levels of IL-2 and TNF- $\alpha$ in S-180 mice and decrease the protein expression of rasP21 and $\mathrm{P} 53$ [34]. AEM polysaccharides inhibit $\mathrm{S} 180$ sarcoma growth in vivo by upregulating the activity of immunocytes[35]. AEM also possesses unique cardioprotective effects. AEM saponins could attenuate the injury induced by $\mathrm{H}_{2} \mathrm{O}_{2}$ in neonatal rat cardiomyocytes through scavenging free radicals, enhancing the activities of antioxidant enzymes, reducing myocardial oxygen consumption, and increasing the stabilization of myocardial cells[36]. Additionally, AEM saponins also has protective effects on ischemia/reperfusion injury through increasing the activity of antioxidant enzyme SOD, reducing the toxic action of oxygen radical to cardiocytes, and inhibiting the NLRP3 pathway[37-38].

\section{Rhaponticum carthamoides}

R. carthamoides (Asteraceae family), commonly known as a maral root or Russian leuzea, is a perennial herb which is mainly distributed in the northern part of Heilongjiang province and Altai and Saian Mountains of South Siberia[39]. Its root and rhizome have been widely used in traditional Siberian medicine as nutraceutical preparations and diet supplements for overstrain and common weakness after illness[40]. R. carthamoides has the effect of invigorating $Q i$ and nourishing the brain and is used for the treatment of anorexia and neurasthenia[41]. In China, R. carthamoides is a folk herbal medicine used as a tonic for improving sexual dysfunction, weakness, and cardiovascular disease[42]. Interestingly, R. carthamoides has also been used by elite Soviet and Russian athletes for its ability to enhance psychological and physical reserves[43]. Modern pharmacological research demonstrated that the extract and compounds isolated from R. carthamoides have beneficial effects, including regulation of immunity, elimination of free radicals, increases of protein biosynthesis and physical work capacity, enhancement of cardiovascular function and mental work capacity, and on the like[42]. Increasing evidence has demonstrated that steroids, particularly ecdysterone, one of the main ecdysteroids in R. carthamoides, by enhancing overall fitness and health[44]. Meanwhile, polysaccharides in $\mathrm{R}$. carthamoides also could prolong swimming time of mice and enhance humoral immunity[45].

In conclusion, Chinese herbs grown in special growth environment have showcased their great potential for applications to the treatment of various diseases due to their diverse functionalities. Not limited to the aforementioned herbs, many other analogous herbs or even new herbs with beneficial effects are yet be discovered. However, the mechanisms and concrete therapeutic usage remain uncovered. Future studies should be orientated to elucidating the chemical material basis responsible for the beneficial effects, mechanism of action, and various pharmacological effects on immunity, cardiovascular function, and anti-cancer effects. It is anticipated that the unique herbs will be broadly and effectively applied to maintaining the human health and treating intractable diseases.

\section{Conflict of interests}

All authors declare no competing financial interest. 


\section{References}

[1] Brekhman I I, Dardymov I V. New substances of plant origin which increase nonspecific resistance. Annu Rev Pharmacol, 1969; 9: 419-430. [2] Panossian A, Brendler T. The role of adaptogens in prophylaxis and treatment of viral respiratory infections. Pharmaceuticals, 2020; 13(9): 236.

[3] Asea A, Kaur P, Panossian A, et al. Evaluation of molecular chaperons Hsp72 and neuropeptide $Y$ as characteristic markers of adaptogenic activity of plant extracts. Phytomedicine, 2013; 20(14): 1323-1329.

[4] Irfan M, Kwak Y S, Han C K, et al. Adaptogenic effects of Panax ginseng on modulation of cardiovascular functions. J Ginseng Res, 2020; 44: 538-543.

[5] Ratan Z A, Youn S H, Kwak Y S, et al. Adaptogenic effects of Panax ginseng on modulation of immune functions. J Ginseng Res, 2020; 45: 32-40.

[6] Patel S, Rauf A. Adaptogenic herb ginseng (Panax) as medical food: status quo and future prospects. Biomed Pharmcother, 2017; 85: 120127.

[7] Yang W Z, Hu Y, Wu W Y, et al. Saponins in the genus Panax L. (Araliaceae): a systematic review of their chemical diversity. Phytochemistry, 2014; 106: 7-24.

[8] Dang H X, Chen Y, Liu X M, et al. Antidepressant effects of ginseng total saponins in the forced swimming test and chronic mild stress models of depression. Prog Neuro-Psychopharmacol Biol Psychiatry, 2009; 33(8): 1417-1424.

[9] Park J S, Park E M, Kim D H, et al. Anti-inflammatory mechanism of ginseng saponins in activated microglia. J Neuroimmunol, 2009; 209(12): 40-49.

[10] Kim J H, Yi Y S, Kim M Y, et al. Role of ginsenosides, the main active components of Panax ginseng, in inflammatory responses and diseases. J Ginseng Res, 2017; 41(4): 435-443.

[11] Nabavi S F, Braidy N, Orhan I E, et al. Rhodiola rosea $L$ and Alzheimer's disease: from farm to pharmacy. Phytother. Res, 2016; 30(4): 532-539.

[12] Zhuang W, Yue L, Dang X, et al. Rosenroot (Rhodiola): potential applications in aging-related diseases. Aging dis, 2019; 10(1): 34-146.

[13] Ma G, Zheng Q, Xu M, et al. Improves learning and memory function: preclinical evidence and possible mechanisms. Front Pharmacol, 2018; 9: 1415.

[14] Pu W, Zhang M, Bai R, et al. Anti-inflammatory effects of Rhodiola rosea L.: a review. Biomed Pharmacother, 2020; 121: 109552.

[15] Amsterasm J D, Panossian A G. Rhodiola rosea L. As a putative botanical antidepressant. Phytomedicine, 2016; 23(7): 770-783.

[16] Kelly G S. Rhodiola rosea: a possible plant adaptogen. Altern. Med Rev, 2001; 6(3): 293-302.

[17] Elameen A, Klemsdal, S S, Dragland, S, et al. Genetic diversity in a germplasm collection of roseroot (Rhodiola rosea) in Norway studied by AFLP. Biochem Syst Ecol, 2008; 36(9): 706-715.

[18] Szopa A, Ekiert R, Ekiert H. Current knowledge of Schisandra chinensis (Turcz.) Baill. (Chinese magnolia vine) as a medicinal plant species: a review on the bioactive components, pharmacological properties, analytical and biotechnological studies. Phytochem Rev, 2017; 16: 195-218.

[19] Xia N, Li J, Wang H, et al. Schisandra chinensis and Rhodiola rosea exert an anti-stress effect on the HPA axis and reduce hypothalamic
c-Fos expression in rats subjected to repeated stress. Exp Ther Med, 2016; 11(1): 353-359.

[20] Nowak A, Zakłos-Szyda M, Błasiak J, et al. Potential of Schisandra chinensis (Turcz.) Baill. in human health and nutrition: a review of current knowledge and therapeutic perspectives. Nutrients, 2019; 11(2): 333.

[21] Szopa A, Barnaś M, Ekiert H. Phytochemical studies and biological activity of three Chinese Schisandra species (Schisandra sphenanthera, Schisandra henryi and Schisandra rubriflora): current findings and future applications. Phytochem Rev, 2019; 18: 109-128.

[22] Jia A L, Zhang $Y H$, Gao H, et al. A review of Acanthopanax senticosus (Rupr and Maxim.) harms: From ethnopharmacological use to modern application. J Ethnopharmacol, 2021; 268: 113586.

[23] Yang J B, Cai W, Li M H, et al. Progress in chemical and pharmacological research of Acanthopanax gracilistylus. Mod Chin Med, 2020; 22(4): 652-662.

[24] China Pharmacopoeia Committee, 2020. 2020 Edition of Chinese Pharmacopoeia. Beijing: China Pharmacopoeia Committee, 215-216.

[25] Zhou Y Y, Song W, Fu Y L, et al. Acanthopanax senticosus reduces brain injury in mice exposed to low linear energy transfer radiation. Biomed Pharmacother, 2018; 99: 781-790.

[26] Sui D Y, Lu Z Z, Ma L N, et al. Effects of the leaves of Acanthopanax senticosus (Rupr. et Maxim.) Harms. on myocardial infarct size in acute ischemic dogs. China J Chin Materia Medica, 1994; 764: 746-747.

[27] Zhong L, Ye Q R. Protective effects of Acanthopanecis senticasi on experimental ischemic myocardium. Chin. J. Pathophysiology, 1995; 11: 366-369.

[28] Liu G H, Zhang P H, Wang W Z, et al. Study on braiding parameters of a biodegradable nerve regeneration conduit with regular braided structure. J Dong Hua Univ, 2004; 6: 66-70.

[29] Sui D Y, Qu S C, Yu X F, et al. Protective effect of ASS on myocardial ischemia-reperfusion injury in rats. China $\mathrm{J}$ Chin Materia Medica. 2004; 29: 71-74.

[30] Sui D Y, Han C C, Yu X F, et al. Effects of Acanthopanax Senticosus saponins on blood lipid metabolism and antioxidative activity in experimental hyperlipidemia rats. J Jilin Univ. (Med Ed), 2004; 30(1): 5659.

[31] Liang Q M, Yu X F, Qu S C, et al. Acanthopanax senticosides B ameliorates oxidative damage induced by hydrogen peroxide in cultured neonatal rat cardiomyocytes. Eur J Pharmacol, 2010; 627(1-3): 209215.

[32] Yang S B, Shan C L, Ma X, et al. Immunomodulatory effect of Acanthopanax senticosus polysaccharide on immunosuppressed chickens. Poult Sci, 2021; 100(2): 623-630.

[33] Song J D, Wu L P, Zhu C X, et al. A review of the pharmacological effects of triterpenoid saponins from Aralia elata. Ginseng Res, 2019; 4: 47-51.

[34] Wu B Y, Han Y Y, Liang Y, et al. The mechanism of action research of TSAS resist tumor of mice. Liaoning J Tradit Chin Med, 2010; 37(1): 175-176.

[35] Wang L J, Jiang $\mathrm{H}$. Antitumor activity of polysaccharide from Aralia elata Seem and the effect on immune functions in tumor-bearing mice. Chin J Immunol, 2011; 27: 130-134.

[36] Sun G B, Wang M, Gao M M, et al. Protective effects of Aralosides (AS) against $\mathrm{H} 2 \mathrm{O} 2$-induced injury in neonatal rat cardiomyocytes. Chin 
Pharmacol Bull, 2013; 29(6): 773-777.

[37] Lu W X, Li M, Wang T. Study of protection mechanism of aralosides on myocardial ischemia/reperfusion injury in rats. China J Tradit Chin Med Pharm, 2011; 26(3): 554-556.

[38] Sun L, Song X L, Yu M, et al. Protective effects and mechanism of total sapoins of Aralia elata on ischemia/reperfusion injury of isolated heart. Global Tradit Chin Med, 2020; 13(2): 207-212.

[39] Lotocka B, Geszprych A. Anatomy of the vegetative organs and secretory structures of Rhaponticum carthamoides (Asteraceae). Bot J Linn Soc, 2004; 144(2): 207-233.

[40] Petkov V, Roussinov K, Todorov S, et al. Pharmacological investigations on Rhaponticum carthamoides. Planta Med, 1984; 50(3): 205-209.

[41] Xu X, Baharguri H, Jia X G. Kazakh Medicinal Records. Urumqi: The Ethnic Publishing House, 2009: 239-241.
[42] Kokoska L, Janovska D. Chemistry and pharmacology of Rhaponticum carthamoides: a review. Phytochemisty, 2009; 70(7): 842855.

[43] Skala E, Synowiec E, Kowalczyk T, et al. Rhaponticum carthamoides transformed root extract has potent anticancer activity in human leukemia and lung adenocarcinoma cell lines. Oxid Med Cell Longev, 2018.

[44] Roumanille R, Vernus B, Brioche T, et al. Acute and chronic effects of Rhaponticum carthamoides and Rhodiola rosea extracts supplementation coupled to resistance exercise on muscle protein synthesis and mechanical power in rats. J Int Soc Sport Nutr, 2020; 17 : 58-70.

[45] Li D M, Fu B, Wei W R, et al. Experimental study on pharmacological action of Polysaccharide from Rhaponticum carthamoides. Pharmacol Clin of Chin Mater Medica, 2000; 16(1): 17-18. 